\title{
Probability functions in the context of signed involutive meadows
}

\author{
Jan A. Bergstra \& Alban Ponse \\ Informatics Institute, University of Amsterdam \\ https://staff.fnwi.uva.nl/j.a.bergstra/ https://staff.fnwi.uva.nl/a.ponse/
}

\begin{abstract}
The Kolmogorov axioms for probability functions are placed in the context of signed meadows. A completeness theorem is stated and proven for the resulting equational theory of probability calculus. Elementary definitions of probability theory are restated in this framework.

Keywords and phrases: Meadow, Bayes' theorem, Bayesian reasoning
\end{abstract}

\section{Introduction}

The Kolmogorov axioms for probability functions may be considered a module that can be included in a variety of more or less formalized contexts. We will propose and investigate some consequences of these axioms when placed in the context of involutive meadows, that is meadows where inverse is an involution following the terminology of [7, 1

In particular we will discuss an axiomatization of a probability function $(\mathrm{PF})$ on a Boolean algebra. The Boolean algebra serves as an event space, the PF defined on it produces elements of (values in) a signed meadow that serve as probabilities. Special focus is on the case where values are chosen in the signed meadow of real numbers. The following objectives motivate the line of development in this paper.

1. To develop an approach towards strictly equational reasoning about probability.

2. To provide a finite loose equational specification of probability functions.

3. To provide a useful completeness result for equational axioms of probability functions.

4. To investigate some total versions of the conditional probability operator.

5. To initiate the development of an application for the theory of signed meadows as outlined in 4 ] and [5].

\footnotetext{
${ }^{1}$ This paper is a revision of arXiv:1307.5173v2. The paper needed to be revised because we found too late that an equation mentioned as an axiom before is in fact derivable from the other axioms as stated. This fact called for a significant change in emphasis and presentation. In addition a survey has been provided of some options to turn conditional probability into a total operator.
} 
We will produce an axiom system consisting of twenty-six equational axioms covering Boolean algebra, meadows, the sign function, and the PF. Then we will introduce several derived operators and prove a number of simple facts, including Bayes' theorem.

These axioms constitute a finite equational basis for the class of Boolean algebra based, real-valued PFs. In other words, the completeness results of [4, 5] extend to the case with Boolean algebra based PFs. We understand this result to convey that the set of twenty-six axioms is complete in a reasonable sense.

The paper is structured as follows: in the remainder of this section we discuss the concept of a meadow in more detail and provide a survey of relevant design options. In Section 2 we introduce some preliminaries. In Section 3 we provide equational axioms for a $\mathrm{PF}$, and in Section 4 we discuss completeness. In Section 5 we consider multi-dimensional probability functions, and Section $[6$ contains some concluding remarks. In Appendix $\mathrm{A}$ we discuss an example of equational probabilistic reasoning.

\subsection{A survey of design options for the inverse of 0}

A meadow is a ring-like structure equipped with an inverse function. A ring based meadow expands a ring with a one place inverse function (inversive notation), or a two place division function (divisive notation). The terms 'inversive notation' and 'divisive notation' were coined in [6].

The key design choice that needs to be made when contemplating a meadow concerns the way it handles the inverse of 0 . In a rather scattered literature on the subject a plurality of different options has been developed and studied, though in varying levels of detail. A brief survey of these endeavours sets the stage for the plan of this paper. The listing below is incomplete, but it contains all proposals for which we have been able to find an unambiguous description. As a criterion regarding this judgement we have required that (i) it must be possible to find out when a closed expressions written using $0,1,+,-, \cdot,(-)^{-1}$ is considered to have a value in the mathematical structure at hand, (ii) for two closed expressions both having a value it must be possible to determine equality in the same structure, and (iii) the relation between inverse and division must be transparent. We will distinguish three design options for ring based meadows and three design options for non-ring based meadows. We will first survey design options for non-ring based meadows.

\section{Non-ring based meadows}

Three options for setting the inverse of zero in a non-ring based meadow stand out, each involving an error value which fails to meet the requirements of a ring. Distinguishing these options is facilitated by making use of a uniform terminology.

Natural inverse. If $0^{-1}$ is equated with an unsigned infinite value, often denoted by $\infty$, then 0 is said to have a natural inverse. The use of natural inverse in mathematics dates back to Riemann at least. Wheels are the prominent instance of meadows with natural inverse, see 9 .

Signed natural inverse. If the inverse of zero is equated with a signed infinite value (written say as $+\infty=\infty$, which differs from $-\infty$ ) we propose to speak of a signed natural inverse. This design choice underlies the transreals and transrationals, see [15]. 
Common inverse. If the inverse of zero is equated with an error value a then, following 8 , zero is said to have a common inverse. Common meadows are meadows based on common inverse. The error value a satisfies $x+\mathbf{a}=x \cdot \mathbf{a}=-\mathbf{a}=\mathbf{a}$ and for that reason fails to comply with the requirements for a ring $(0 \cdot x=0)$. Moreover, the error value is unique.

Also in the case of natural inverse and signed natural inverse, the error value(s) fail to comply with the requirements for a ring $(0 \cdot x=0)$.

\section{Ring based meadows}

For ring based meadows three options may be distinguished.

Partial inverse. The most prominent ring based meadow leaves the inverse of 0 undefined and considers inverse to be a partial function.

Working with partial inverse deviates from mathematical practice to the extent that questions like whether or not $1 / 0=2 / 0$ must be taken seriously. When dealing with partial inverse there are no semantic questions about it, but the choice of a logic of partial functions leaves substantial room for design variation, beginning with a choice between three ways of looking at the truth value of say $1 / 0=1 / 0$ : is it considered as being true, or as being false in an overarching two-valued logic, or as not being true in an overarching logic which is not two-valued.

Symmetric inverse. If the meadow is based on a regular ring and the value of $0^{-1}$ is taken to be 0,0 is said to have a symmetric inverse. The meadows of [4, 5] and several preceding papers are ring based meadows with symmetric inverse. Alternatively this case is referred to as featuring an involutive inverse, and such meadows are referred to as involutive meadows.

Non-involutive inverse. If the inverse of $0^{-1}$ is taken to be different from $0,\left(x^{-1}\right)^{-1}=x$ cannot hold, that is inverse is not an involution, and inverse is said to be non-involutive. The non-involutive meadows discussed in 7 that satisfy $0^{-1}=1$ are ring based meadows with an asymmetric inverse. If the inverse of $0^{-1}$ is taken to be say 17 or any (rational or real) number different from 0 and 1, 0 is said to have an ad hoc non-involutive inverse. Ad hoc non-involutive inverses come into play when formalizing the theory of fields in first order logic in the presence of a function symbol for either inverse or division (or both).

\subsection{Working with involutive ring based meadows}

In this paper we will work exclusively with ring based involutive meadows, which will be referred to simply as meadows. The motivation for this choice is that it appears to be a most straightforward way to pursue the objectives that were listed above. However, we do not claim that for the purpose of developing an equational approach to probability working with ring based meadows is the best option, neither do we claim that among the three options for ring based meadows working with a symmetric inverse is best suited to this objective. 


$$
\begin{aligned}
(x \vee y) \wedge y & =y \\
(x \wedge y) \vee y & =y \\
x \wedge(y \vee z) & =(y \wedge x) \vee(z \wedge x) \\
x \vee(y \wedge z) & =(y \vee x) \wedge(z \vee x) \\
x \wedge \neg x & =\perp \\
x \vee \neg x & =\top
\end{aligned}
$$

Table 1: $B A$, a self-dual equational basis for Boolean algebras

\section{Boolean algebras and meadows}

In this section we specify the mathematical context on which our axiomatization is based. In particular, we provide specifications for Boolean algebras (Section 2.2), and for events and (signed) meadows (Section 2.3).

\subsection{Boolean algebras}

A Boolean algebra $\left(B,+, \cdot,^{\prime}, 1,0\right)$ may be defined as a system with at least two elements such that $\forall x, y, z \in B$ the well-know postulates of Boolean algebra are valid. Because we want to avoid overlap with the operations of a meadow, we will consider Boolean algebras with notation from propositional logic, thus consider $(B, \vee, \wedge, \neg, \top, \perp)$ and adopt the axioms in Table 1. In [13] it was shown that the axioms in Table 1 constitute an equational basis.

\subsection{Valuated Boolean algebras and some naming conventions}

A Boolean algebra can be equipped with a valuation $v$ that assigns to its elements values in a signed meadow.

In this paper we will investigate the special case where the valuation function of a valuated Boolean algebra is a probability function by requiring that the valuation satisfies the Kolmogorov axioms for probability functions cast to the setting of signed meadows.

By way of notational convention we will from now on assume that $E$ (for events) is the name of the carrier of a Boolean algebra, and that $V$ (for values) names the carrier of the meadow in a valuated Boolean algebra.

\section{$2.3 \quad$ Events and signed meadows}

The set of axioms in Table 2 specifies the class of meadows.

In the setting of probability functions the elements of the underlying Boolean algebra are referred to as events 2 We will use "value" to refer to an element of a meadow 3 and a

\footnotetext{
${ }^{2}$ Events are closed under $-\vee-$, which represents alternative occurrence and $-\wedge-$, which represents simultaneous occurrence, and under negation.

${ }^{3}$ Rational numbers and real numbers are instances of values.
} 


$$
\begin{aligned}
(x+y)+z & =x+(y+z) \\
x+y & =y+x \\
x+0 & =x \\
x+(-x) & =0 \\
(x \cdot y) \cdot z & =x \cdot(y \cdot z) \\
x \cdot y & =y \cdot x \\
1 \cdot x & =x \\
x \cdot(y+z) & =x \cdot y+x \cdot z \\
\left(x^{-1}\right)^{-1} & =x \\
x \cdot\left(x \cdot x^{-1}\right) & =x
\end{aligned}
$$

Table 2: $M d$, a set of axioms for meadows

probability function is a valuation (from events to the values in a signed meadow) 4

An expression of type $E$ is an event expression or an event term, an expression of type $V$ is a value expression or equivalently a value term. In the signature of a valuated Boolean algebra there is just one notation for a probability function, the function symbol $P 5$

The axioms in Table 3 specify the sign function $\mathbf{s}(-)$, which presupposes an ordering $<$ on its domain and is defined by

$$
\mathbf{s}(x)= \begin{cases}-1 & \text { if } x<0 \\ 0 & \text { if } x=0 \\ 1 & \text { if } 0<x\end{cases}
$$

Before commenting on these axioms, we introduce some abbreviations. First, we shall further write $x-y$ for $x+(-y)$. Below we define the conditional expression $p \triangleleft q \triangleright r$, two notations for a division operator, absolute value, and orderings, where $p, q$ and $r$ range over $V$, the carrier of the signed meadow in a valuated Boolean algebra.

1. $1_{p}={ }_{\operatorname{def}} p \cdot p^{-1}$,

2. $0_{p}={ }_{\text {def }} 1-1_{p}$,

3. $p \triangleleft q \triangleright r==_{\text {def }} 1_{q} \cdot p+0_{q} \cdot r$,

4. $\frac{p}{q}={ }_{\text {def }} p \cdot q^{-1}$,

5. $p / q={ }_{\operatorname{def}} \frac{p}{q}$,

\footnotetext{
${ }^{4}$ We will exclude probability functions with negative values, a phenomenon known in non-commutative probability theory, leaving the exploration of that kind of generalization to future work.

${ }^{5}$ In some cases the restriction to a single probability function $P$ is impractical and providing a dedicated sort for such functions brings more flexibility and expressive power. This expansion may be achieved in different ways.
} 


$$
\begin{aligned}
\mathbf{s}\left(1_{x}\right) & =1_{x} \\
\mathbf{s}\left(0_{x}\right) & =0_{x} \\
\mathbf{s}(-1) & =-1 \\
\mathbf{s}\left(x^{-1}\right) & =\mathbf{s}(x) \\
\mathbf{s}(x \cdot y) & =\mathbf{s}(x) \cdot \mathbf{s}(y) \\
0_{\mathbf{s}(x)-\mathbf{s}(y)} \cdot(\mathbf{s}(x+y)-\mathbf{s}(x)) & =0
\end{aligned}
$$

Table 3: Sign, a set of axioms for the sign operator

6. $|p|=_{\text {def }} \mathbf{s}(p) \cdot p$,

7. $p<q==_{\text {def }} \mathbf{s}(q-p)=1$, and

8. $p \leq q={ }_{\text {def }} \mathbf{s}(\mathbf{s}(q-p)+1)=1$.

In Table 3, axiom (22) is an equational representation of the conditional equation

$$
\mathbf{s}(x)=\mathbf{s}(y) \rightarrow \mathbf{s}(x+y)=\mathbf{s}(x) .
$$

This can be seen as follows: if $\mathbf{s}(x)=\mathbf{s}(y)$, then $0_{\mathbf{s}(x)-\mathbf{s}(y)}=1$ and $(\mathbf{s}(x+y)-\mathbf{s}(x))=0$, and if $\mathbf{s}(x) \neq \mathbf{s}(y)$ then $0_{\mathbf{s}(x)-\mathbf{s}(y)}=0$.

Together, Tables 2 and 3 contain the axioms $M d+$ Sign for signed meadows (we write + instead of $\cup$ ). In 4 the following identities were shown to be consequences of $M d+$ Sign:

$$
\begin{array}{ll}
\mathbf{s}\left(x^{2}\right)=1_{x} & \mathbf{s}(x)^{-1}=\mathbf{s}(x) \\
\mathbf{s}\left(x^{3}\right)=\mathbf{s}(x) & \mathbf{s}(\mathbf{s}(x))=\mathbf{s}(x)
\end{array}
$$

We further note that the equivalence $\mathbf{s}(\mathbf{s}(p)+1)=1 \Longleftrightarrow p=\mathbf{s}(p) \cdot p=|p|$ is provable from $M d+$ Sign (this follows easily from Theorem 4.1.1 below).

We will also consider the subclass of signed cancellation meadows. A cancellation meadow satisfies the Inverse Law (IL) of Table 4.

\section{Signed meadow based probability calculus}

In Section 3.1 we formulate axioms for a probability function. Following the methods of abstract data type specification we will focus on axioms in equational form. Then, we discuss a plurality of versions of the conditional probability operator (Section 3.2) and some properties thereof, in particular versions of Bayes' theorem. Finally, we consider independent events (Section 3.31).

$$
x \neq 0 \longrightarrow x \cdot x^{-1}=1 .
$$

Table 4: Inverse Law (IL) 


$$
\begin{aligned}
P(\top) & =1 \\
P(\perp) & =0 \\
P(x) & =|P(x)| \\
P(x \vee y) & =P(x)+P(y)-P(x \wedge y)
\end{aligned}
$$

Table 5: $P F_{P}$, a set of axioms for a probability function with name $P$

\subsection{Equational axioms for a probability function}

In Table 5 we define the set $P F_{P}$ of axioms for a probability function. These axioms represent Kolmogorov's axioms in the context of a Boolean algebra (rather than a universe of sets) and a signed meadow (instead of a field). Axiom (25) expresses that the sign of $P(x)$ is nonnegative. Axiom (26) distributes $P$ over finite unions. In the absence of an infinitary version of axiom (26) we consider these axioms to constitute an axiomatization for the restricted concept of probability functions only, rather than for probability measures in general.

In combination with the axioms $B A+M d$, the two axioms (24) and (26) in Table 5 can be replaced by the single axiom

$$
P(x)=P(x \wedge y)+P(x \wedge \neg y)
$$

where the expressions $x \wedge y$ and $x \wedge \neg y$ characterize two disjoint (mutually exclusive) events: axiom (24) follows from $P(x)=P(x \wedge x)+P(x \wedge \neg x)$, thus $P(x \wedge \neg x)=P(\perp)=0$, and axiom (26) follows from

$$
P(x \vee y) \stackrel{\boxplus}{\boxplus} P((x \vee y) \wedge x)+P((x \vee y) \wedge \neg x)=P(x)+P(y \wedge \neg x)
$$

and $P(y) \stackrel{\boxplus}{\boxplus} P(y \wedge x)+P(y \wedge \neg x)$, thus $P(y \wedge \neg x)=P(y)-P(x \wedge y)$. Conversely, axiom $\boxplus$ ) follows from (24) and (26):

$$
P(x)=P((x \wedge y) \vee(x \wedge \neg y))=P(x \wedge y)+P(x \wedge \neg y)-P(\perp) .
$$

A valuated Boolean algebra equipped with a valuation $P$ in some signed meadow $\mathbb{M}$ with carrier $V$ that satisfies all axioms of

$$
B A+M d+\operatorname{Sign}+P F_{P}
$$

will be called a $K(\mathbb{M}, P)$-structure.

Theorem 3.1.1 (Disjoint event factorization). $B A+M d+P F_{P} \vdash P(x)=P(x \wedge y)+P(x \wedge \neg y)$.

Proof. This is $($, which is shown above.

Theorem 3.1.2 (Probability upper bound). $B A+M d+S i g n+P F_{P} \vdash P(x) \leq 1$.

Proof. First notice $1=P(\top)=P(x \vee \neg x)=(P(x)+P(\neg x))-P(\perp)=P(x)+P(\neg x)$, so $P(x)=1-P(\neg x)$. Because $P(\neg x) \geq 0$ we conclude $P(x) \leq 1$. 
The following theorem asserts in equational form the conditional equation $P(y)=0 \rightarrow$ $P(x \wedge y)=0$, using inversive notation.

Theorem 3.1.3. $B A+M d+\operatorname{Sign}+P F_{P} \vdash P(x \wedge y) \cdot P(y) \cdot P(y)^{-1}=P(x \wedge y)$.

Proof. Let $\phi(u, v)$ be as follows:

$$
\phi(u, v) \equiv\left(1-\frac{|u|+|v|}{|u|+|v|}\right) \cdot u
$$

Now $\left(\mathbb{R}_{0}, \mathbf{s}\right) \models \phi(u, v)=0$, and using the completeness theorem of [5] one obtains $M d+\operatorname{Sign} \vdash$ $\phi(u, v)=0$. Substituting $P(y \wedge x)$ for $u$ and $P(y \wedge \neg x)$ for $v$ and applying Theorem 3.1.1, one derives

$$
\begin{aligned}
B A+M d+\operatorname{Sign}+P F_{P} \vdash 0 & =\left(1-\frac{|P(y \wedge x)|+|P(y \wedge \neg x)|}{|P(y \wedge x)|+|P(y \wedge \neg x)|}\right) \cdot P(y \wedge x) \\
& =\left(1-\frac{P(y)}{P(y)}\right) \cdot P(y \wedge x),
\end{aligned}
$$

from which the required result follows immediately.

\subsection{Conditional probability as a total operator: four options}

Conditional probability $P(x \mid y)$ of event $x$ relative to event $y$ is conventionally understood as a partial function of $x$ and $y$, defined only if $P(y)$ is nonzero. The objective of developing an equational logic for probability theory suggests that total versions of the conditional probability operator ought to be contemplated.

Conditional probability defined according to Kolmogorov is written below as $P^{\star}(x \mid y)$, where variables $x$ and $y$ range over $E$, and is defined by

$$
P^{\star}(x \mid y)=_{\text {def }} \frac{P(x \wedge y)}{P(y)} \triangleleft P(y) \triangleright \uparrow .
$$

Here $\uparrow$ denotes that the result is undefined 6 The key advantage of partial conditional probability is that one does not introduce a value for, say $P(x \mid \perp)$ which might be subsequently disputed.

Four ways of making conditional probability defined on all inputs will now be distinguished.

Definition 3.2.1 (Zero-totalized conditional probability). $\quad P^{0}(x \mid y)=_{\operatorname{def}} \frac{P(x \wedge y)}{P(y)}$.

\footnotetext{
${ }^{6}$ We assume that in a context of partial functions an identity $t=r$ is valid if either both sides are undefined or both sides are defined and equal. This convention, however, leaves room for alternative readings of the expressions at hand. In particular the definition given for $x \triangleleft y \triangleright z$ implies that whenever $t$ is undefined, so is $t \triangleleft r \triangleright s$. That is not a very plausible feature of the conditional and in the presence of partial operations the conditional operator requires a different definition. These complications are to some extent avoided, or rather made entirely explicit, when working with total functions. The use of the notation $P^{\star}(-\mid-)$ instead of the common notation $P(-\mid-)$ is justified by the fact that unavoidably $P^{\star}(-\mid-)$ inherits properties from the equational specification of the functions from which it has been made up. Such properties need not not coincide with what is expected from $P(-\mid-)$.
} 
We notice that $P^{0}(T \mid \perp)=0$, a choice for which no convincing philosophical motivation can be put forward. Two advantages can be put forward in favour of $P^{0}(-\mid-)$ : the logical simplicity that comes with it being total and the calculational simplicity that comes with choosing 0 as a value for $P^{0}(x \mid y)$ when $P(y)=0$. The following properties are immediate:

$$
P^{0}(x \mid x)=\frac{P(x)}{P(x)} \quad \text { and } \quad P(x)=P(x) \cdot P^{0}(x \mid x) .
$$

Moreover we have 'joint probability factorization':

$$
P(x \wedge y)=P(x \wedge y) \cdot P(y) \cdot P(y)^{-1}=(P(x \wedge y) / P(y)) \cdot P(y)=P^{0}(x \mid y) \cdot P(y),
$$

and 'total probability':

$$
\begin{aligned}
P(x) & =P(x \wedge y)+P(x \wedge \neg y) \\
& =P(x \wedge y) \cdot P(y) \cdot P(y)^{-1}+P(x \wedge \neg y) \cdot P(\neg y) \cdot P(\neg y)^{-1} \\
& =P^{0}(x \mid y) \cdot P(y)+P^{0}(x \mid \neg y) \cdot P(\neg y) .
\end{aligned}
$$

As another illustration of the latter advantage we provide below a proof of Bayes' rule in its simplest form.

Definition 3.2.2 (One-totalized conditional probability). $\quad P^{1}(x \mid y)=_{\operatorname{def}} \frac{P(x \wedge y)}{P(y)} \triangleleft P(y) \triangleright 1$.

We will write $x \rightarrow y$ for $\neg x \vee y$. The principal advantage of one-totalized conditional probability over zero-totalized conditional probability is the validity of the following rule, which provides some intrinsic motivation for this design of conditional probability:

$$
(x \rightarrow y)=\top \Rightarrow P^{1}(x \mid y)=1 .
$$

If $\alpha \in\{\star, 0,1\}$ then the function

$$
P \circ^{\alpha} y=_{\text {def }} \lambda x \in E \cdot P^{\alpha}(x \mid y)
$$

is not a probability function for each $y$. In particular, if $P(y)=0, P \circ^{\alpha} y$ will fail to comply with either $P \circ^{\alpha} y(\top)=1$ or with $P \circ^{\alpha} y(\perp)=0$. Now $\lambda P \in P F . P \circ^{\alpha} y$ being the wellknown update operator that goes with some applications of Bayes' theorem, it is a reasonable requirement that this very operator becomes total as well. We will introduce two options for conditionalization which achieve this requirement.

Definition 3.2.3 (Safe conditional probability). $\quad P^{s}(x \mid y)=_{\text {def }} \frac{P(x \wedge y)}{P(y)} \triangleleft P(y) \triangleright P(x)$.

We find that $P \circ^{s} y=P$ if $P(y)=0$, which allows the view that $\lambda P . P \circ^{s} y$ is an operator mapping probability functions to probability functions for all events $y$, or stated differently that $\lambda y .\left(\lambda P . P \circ^{s} y\right)$ is a total mapping from events to probability function transformations. $P \circ^{s}$ is safe because it enforces no update when an inconsistency is observed.

Yet another way to achieve this property of a conditional update is to return an exceptional value, in this case the canonical probability function for an atomic event. An atom in $E$ is an 
event $a \in E$ which satisfies atom $(a)=_{\text {def }} \forall x \in E .(x \wedge a=a$ OR $x \wedge a=\perp)$. For an atom $a \in E$ the probability function $\mathrm{pf}_{a}$ is defined by:

$$
\operatorname{pf}_{a}(x)={ }_{\operatorname{def}} \begin{cases}1 & \text { if } x \wedge a=a, \\ 0 & \text { if } x \wedge a=\perp .\end{cases}
$$

Definition 3.2.4 (Exception raising conditional probability for $a \in E$ ).

$$
P^{e / a}(x \mid y)=_{\operatorname{def}} \frac{P(x \wedge y)}{P(y)} \triangleleft P(y) \triangleright \operatorname{pf}_{a}(x) .
$$

For $P^{0}(-\mid-), P^{s}(-\mid-)$, and $P^{e / a}(-\mid-)$ we are not aware of earlier definitions, whereas $P^{1}(-\mid-)$ has been considered by Adams in [1, and in subsequent literature. For a survey of conditional logic and conditional probabilities we refer to [11].

Of particular importance given its ubiquitous use is Bayes' theorem. Bayes' theorem takes different forms for different versions of conditional probability and in each of these cases it appears as a consequence of $B A+M d+S i g n+P F_{P}$.

Theorem 3.2.5 (Versions of Bayes' theorem). In $B A+M d+S i g n+P F_{P}$ the following equations are derivable:

1. $P^{0}(x \mid y)=\frac{P^{0}(y \mid x) \cdot P(x)}{P(y)}$

(Bayes' theorem for $P^{0}(-\mid-)$ ),

2. $P^{1}(x \mid y)=\frac{P^{1}(y \mid x) \cdot P(x)}{P(y)} \triangleleft P(y) \triangleright 1$

(Bayes' theorem for $P^{1}(-\mid-)$ ),

3. $P^{s}(x \mid y)=\frac{P^{s}(y \mid x) \cdot P(x)}{P(y)} \triangleleft P(y) \triangleright P(x)$

(Bayes' theorem for $P^{s}(-\mid-)$ ),

4. $P^{e / a}(x \mid y)=\frac{P^{e / a}(y \mid x) \cdot P(x)}{P(y)} \triangleleft P(y) \triangleright \mathrm{pf}_{a}(x)$

(Bayes' theorem for $P^{e / a}(-\mid-)$ ).

Proof. Version 1 can be shown as follows:

$$
\begin{array}{rlr}
P^{0}(x \mid y) & =\frac{P(x \wedge y)}{P(y)} & \\
& =\frac{P(y \wedge x)}{P(y)} \cdot \frac{P(x)}{P(x)} & \text { by Theorem 3.1.3 } \\
& =\frac{P(y \wedge x)}{P(x)} \cdot \frac{P(x)}{P(y)} & \\
& =\frac{P^{0}(y \mid x) \cdot P(x)}{P(y)} .
\end{array}
$$


Version 2 can be shown as follows:

$$
\begin{aligned}
P^{1}(x \mid y) & =\frac{P(x \wedge y)}{P(y)} \triangleleft P(y) \triangleright 1 \\
& =\frac{P(y)}{P(y)} \cdot \frac{P(x \wedge y)}{P(y)}+\left(1-\frac{P(y)}{P(y)}\right) \\
& =\frac{P(y)}{P(y)} \cdot \frac{\left(\frac{P(y \wedge x) \cdot P(x)}{P(x)}\right)}{P(y)}+\left(1-\frac{P(y)}{P(y)}\right) \quad \text { by Theorem } 3.1 .3 \\
& =\frac{P(y)}{P(y)} \cdot \frac{\left(\frac{P(y \wedge x)}{P(x)} \triangleleft P(x) \triangleright 1\right) \cdot P(x)}{P(y)}+\left(1-\frac{P(y)}{P(y)}\right) \\
& =\frac{P(y)}{P(y)} \cdot \frac{P^{1}(y \mid x) \cdot P(x)}{P(y)}+\left(1-\frac{P(y)}{P(y)}\right) \\
& =\frac{P^{1}(y \mid x) \cdot P(x)}{P(y)} \triangleleft P(y) \triangleright 1 .
\end{aligned}
$$

Versions 3 and 4, thus Bayes' theorem for the cases $P^{s}(-\mid-)$ and for $P^{e / a}(-\mid-)$, involve similar calculations and are left to the reader.

\subsection{Independence of events}

Given a $K(\mathbb{M}, P)$-structure, two events $x$ and $y$ are said to be independent relative to that structure if $P(x \wedge y)=P(x) \cdot P(y)$ is valid.

Theorem 3.3.1. Events $x$ and $y$ are independent if and only if $P^{0}(x \mid y)=P(x) \cdot P^{0}(y \mid y)$ and equivalently if and only if $P^{0}(y \mid x)=P(y) \cdot P^{0}(x \mid x)$.

Proof. If $x$ and $y$ are independent, then

$$
P^{0}(x \mid y)=P(x \wedge y) / P(y)=(P(x) \cdot P(y)) / P(y)=P(x) \cdot P^{0}(y \mid y),
$$

and similarly one finds $P^{0}(y \mid x)=P(y) \cdot P^{0}(x \mid x)$.

Conversely, from $P^{0}(x \mid y)=P(x) \cdot P^{0}(y \mid y)$ one finds $P(x \wedge y) / P(y)=P(x) \cdot(P(y) / P(y))$, so multiplying both sides by $P(y)$ yields

$$
P(x \wedge y) \cdot(P(y) / P(y))=P(x) \cdot(P(y) / P(y)) \cdot P(y),
$$

which implies $P(x \wedge y)=P(x) \cdot P(y)$ by Theorem 3.1.3.

\section{Logical aspects of equations for probability functions}

In this section we provide a completeness result for $B A+M d+\operatorname{Sign}+P F_{P}$ (Section 4.1) and discuss the use of a free Boolean algebra as an event space (Section 4.2). 


\subsection{Completeness of $\boldsymbol{B A}+\boldsymbol{M d}+\boldsymbol{S i g n}+\boldsymbol{P} \boldsymbol{F}_{P}$}

In [4] it is shown that $M d+$ Sign constitutes a finite basis for the equational theory of signed cancellation meadows. Stated differently: for each equation $t=r$, if $M d+\operatorname{Sign}+P F_{P}+I L \models$ $t=r$ then also $M d+\operatorname{Sign}+P F_{P} \vdash t=r$. This fact is understood as a completeness results because a stronger set of axioms would necessarily exclude some meadows that are expansions of ordered fields. In a preceding version of this paper $7^{7}$ it was shown that the basis theorem extends to the setting with probability functions: if $B A+M d+S i g n+P F_{P}+I L \models t=r$ then also $B A+M d+\operatorname{Sign}+P F_{P} \vdash t=r$.

For the purposes of this paper we prefer to make use of a different completeness result for the same equational theory that allows us to obtain a more intuitively appealing completeness result for the axiom system $B A+M d+\operatorname{Sign}+P F_{P}$. This second completeness result is given in terms of validity of equations relative to a single signed meadow rather than in an elementary class of structures.

We recall the following result from [5, Thm.3.14], where we write $\mathbb{R}_{0}$ for the meadow that is the expansion of the field of real numbers $\mathbb{R}$ with total inverse operator and $0^{-1}=0$, and $\left(\mathbb{R}_{0}, \mathbf{s}\right)$ for $\mathbb{R}_{0}$ expanded with the sign function $\mathbf{s}(-)$.

Theorem 4.1.1. For an equation $t=r$ in the signature of signed meadows: $\left(\mathbb{R}_{0}, \mathbf{s}\right) \models t=r$ if and only if $M d+$ Sign $\vdash t=r$.

The same completeness result works for conditional equations:

Theorem 4.1.2. For a conditional equation $t_{1}=r_{1} \wedge \ldots \wedge t_{n}=r_{n} \rightarrow t=r$ in the signature of signed meadows: $\left(\mathbb{R}_{0}, \mathbf{s}\right) \models t_{1}=r_{1} \wedge \ldots \wedge t_{n}=r_{n} \rightarrow t=r$ if and only if

$$
M d+\operatorname{Sign} \vdash t_{1}=r_{1} \wedge \ldots \wedge t_{n}=r_{n} \rightarrow t=r .
$$

Proof. The only if part is follows from the soundness of equational logic. If

$$
\left(\mathbb{R}_{0}, \mathbf{s}\right) \models t_{1}=r_{1} \wedge \ldots \wedge t_{n}=r_{n} \rightarrow t=r
$$

then

$$
\left(\mathbb{R}_{0}, \mathbf{s}\right) \models\left(1-\left(t_{1}-r_{1}\right) \cdot\left(t_{1}-r_{1}\right)^{-1}\right) \cdot \ldots \cdot\left(1-\left(t_{n}-r_{n}\right) \cdot\left(t_{n}-r_{n}\right)^{-1}\right) \cdot(t-r)=0 .
$$

Using the above completeness theorem for equational logic with respect to $\mathbb{R}_{0}$, this fact (say $\Phi)$ is provable from $M d+\operatorname{Sign}$. Now assuming that $t_{1}=r_{1} \wedge \ldots \wedge t_{n}=r_{n}$ one finds that

$$
\left(1-\left(t_{1}-r_{1}\right) \cdot\left(t_{1}-r_{1}\right)^{-1}\right) \cdot \ldots \cdot\left(1-\left(t_{n}-r_{n}\right) \cdot\left(t_{n}-r_{n}\right)^{-1}\right)=1
$$

and in combination with $\Phi$ it follows that $t-r=0$.

A $K\left(\mathbb{R}_{0}, P\right)$-structure is a model of $B A+M d+\operatorname{Sign}+P F_{P}$ that contains the meadow of signed reals, $\left(\mathbb{R}_{0}, \mathbf{s}\right)$, as the domain of its values. We will write $K\left(\mathbb{R}_{0}, P\right)$ for the class of $K\left(\mathbb{R}_{0}, P\right)$-structures.

Theorem 4.1 .1 can be extended to the setting of $K\left(\mathbb{R}_{0}, P\right)$-structures thus obtaining a satisfactory completeness result for $B A+M d+\operatorname{Sign}+P F_{P}$.

\footnotetext{
7 http://arxiv.org/abs/1307.5173v1
} 
Theorem 4.1.3. The axiom system $B A+M d+S i g n+P F_{P}$ is sound and complete for the equational theory of $K\left(\mathbb{R}_{0}, P\right) 8$

Proof. Soundness is obvious and therefore we will focus on completeness. First, a valid equation over the sort of Booleans is provable from $B A$ because there are no other means to arrive at the validity of such an equation (no functions from meadows to Booleans).

Next, let $t=r$ be a valid equation over the sort of meadows. Assume $x_{1}, \ldots, x_{k}$ are the variables ranging over $E$ that occur in $t=r$, and $y_{1}, \ldots, y_{\ell}$ are the meadow variables that occur in $t=r$.

Let $m$ be the number of occurrences of $P$ in the equation $t=r$. We list these occurrences in a linear order as $P\left(f_{1}\left(x_{1}, \ldots, x_{k}\right)\right), \ldots, P\left(f_{m}\left(x_{1}, \ldots, x_{k}\right)\right)$ with $f_{i}$ appropriately chosen Boolean expressions over the event variables $x_{1}, \ldots, x_{k}$.

We choose new variables $z_{1}, \ldots, z_{m}$ ranging over $V$, the domain of $\left(\mathbb{R}_{0}, \mathbf{s}\right)$, and we define $t^{\prime}$ and $r^{\prime}$ by replacing in $t$ and $r$ each occurrence of $P\left(f_{i}\left(x_{1}, \ldots, x_{k}\right)\right)$ by $z_{i}$ for $1 \leq i \leq m$.

For $x_{1}, \ldots, x_{k}$ there are $2^{k}$ different conjunctions $\bigwedge_{j=1}^{k} x_{j}^{\prime}$ with $x_{j}^{\prime} \in\left\{x_{j}, \neg x_{j}\right\}$. Let $\alpha_{j}$ be an enumeration of Boolean expressions for these conjunctions $\left(1 \leq j \leq 2^{k}\right)$ and note that

$$
B A \vdash \bigvee_{j=1}^{2^{k}} \alpha_{j}=\top .
$$

We choose $2^{k}$ new variables $u_{j}\left(1 \leq j \leq 2^{k}\right)$ ranging over $V$. We intend to use these variables for representing the values of $P\left(\alpha_{j}\right)$.

Each expression $f_{i}\left(x_{1}, \ldots, x_{k}\right)$ for $1 \leq i \leq m$ can be written as a disjunctive normal form by means of a disjunction of expressions of the form $\alpha_{j}$ :

$$
f_{i}\left(x_{1}, \ldots, x_{k}\right)=\bigvee_{j \in H_{i}} \alpha_{j}
$$

for appropriately chosen subsets $H_{i}$ of $\left\{1, \ldots, 2^{k}\right\}$.

We will now collect $2^{k}+m+1$ equations that together precisely capture the relation between the variables $z_{i}$ and $u_{j}$ under the assumption that for some PF $P, z_{i}=P\left(f_{i}\left(x_{1}, \ldots, x_{k}\right)\right)$ and $u_{j}=P\left(\alpha_{j}\right)$ in some $K\left(\mathbb{R}_{0}, P\right)$-structure. These equations are:

- $u_{j}=\mathbf{s}\left(u_{j}\right) \cdot u_{j}$ for $1 \leq j \leq 2^{k}$, expressing that the $u_{j}$ are nonnegative.

- $z_{i}=\sum_{j \in H_{i}} u_{j}$ for $1 \leq i \leq m$, expressing the consequences in terms of probabilities of the mentioned disjunctive normal form for the expressions $f_{i}\left(x_{1}, \ldots, x_{k}\right)$ in equations $\left(e q_{i}\right)$.

- $\sum_{j=1}^{2^{k}} u_{j}=1$, expressing the fact that $\sum_{j=1}^{2^{k}} P\left(\alpha_{j}\right)=1$, which in turn follows from (园).

We write $\Phi$ for the conjunction of these equations.

Claim. If $B A+M d+\operatorname{Sign}+P F_{P} \models t=r$ then $\left(\mathbb{R}_{0}, \mathbf{s}\right) \models \Phi \rightarrow t^{\prime}=r^{\prime}$.

Proof of Claim. Assume that $B A+M d+\operatorname{Sign}+P F_{P} \models t=r$. Suppose that $\sigma$ is a valuation of the variables $z_{i}, u_{j}, y_{\ell}$ such that $\left(\mathbb{R}_{0}, \mathbf{s}\right), \sigma \models \Phi$. Now from the interpretations of the $z_{i}$ and $u_{j}$ one may construct a Boolean algebra with the $\alpha_{j}$ as generators as well as a PF $P$ on that Boolean algebra. In this structure $t=r$ must hold by assumption, and then $t^{\prime}=r^{\prime}$ follows by substitution of variables for expressions according to the definition of $t^{\prime}$ and $r^{\prime}$. Hence $\left(\mathbb{R}_{0}, \mathbf{s}\right), \sigma \models t^{\prime}=r^{\prime}$. End proof of Claim.

\footnotetext{
${ }^{8}$ More generally, $B A+M d+\operatorname{Sign}+P F_{P}$ is sound for the class of $K(\mathbb{M}, P)$-structures with $\mathbb{M}$ a cancellation meadow.
} 
The completeness result follows from this claim. If $\left(\mathbb{R}_{0}, \mathbf{s}\right) \models \Phi \rightarrow t^{\prime}=r^{\prime}$ then Theorem 4.1.2 implies that this conditional equation can be proven from $M d+\operatorname{Sign}$. The substitution $\theta$ replacing the variables $z_{i}$ by $P\left(f_{i}\left(x_{1}, \ldots, x_{k}\right)\right)$ and $u_{j}$ by $P\left(\alpha_{j}\right)$ can then be applied. Because the axioms of $B A+M d+\operatorname{Sign}+P F_{P}$ suffice to prove that $\theta(\Phi)$ holds, and $\theta\left(t^{\prime}\right) \equiv t$ and $\theta\left(r^{\prime}\right) \equiv r$ with $\equiv$ denoting syntactic equivalence, it follows that $B A+M d+S i g n+P F_{P} \vdash t=r$.

\subsection{Using free Boolean algebras as event spaces}

For the purpose of reformulating some elementary aspects of probability theory and statistics the generality of working with arbitrary Boolean algebras is inessential, at least at this initial stage in the development of an equational callus of probabilities. For that reason we will now introduce several simplifying assumptions:

- A finite set $C$ of constants for events is provided. Elements of $C$ are called primitive events. We will only consider free Boolean algebras generated by the primitive events.

- With $B A_{C}$ we will denote the equations for Boolean algebra in a signature which is expanded with the constants in $C$.

- The class of models of $B A_{C}+M d+\operatorname{Sign}+P F_{P}$ with a free event space over $C,\left(\mathbb{R}_{0}, \mathbf{s}\right)$ as its meadow of values, and a probability function $P$ is denoted $K_{C}\left(\mathbb{R}_{0}, P\right)$. Different structures in $K_{C}\left(\mathbb{R}_{0}, P\right)$ only differ in the choice (interpretation) of the probability function $P$.

These assumptions correspond to what is needed for the specification of examples of probabilistic reasoning.

Theorem 4.2.1. $M d+$ Sign $+B A_{C}+P F_{P}$ is sound and complete for the equations of type $V$ that are true in all structures in $K_{C}\left(\mathbb{R}_{0}, P\right)$. In other words, for $t$ and $r$ terms of sort $V$ :

$$
M d+\text { Sign }+B A_{C}+P F_{P} \vdash t=r \text { if and only if } K_{C}\left(\mathbb{R}_{0}, P\right) \models t=r .
$$

Proof. The proof is merely a reformulation of the proof of Theorem 4.1.3.

\section{Multi-dimensional probability functions}

In this section we provide axioms for multi-dimensional PFs (Section 5.1), and discuss two elementary issues: a condition for the existence of a particular universal PF (Section 5.2), and the relation between the multi-dimensional and the one-dimensional case (Section 5.3).

\subsection{Equational axioms for a probability function family}

Let $D=\left\{a_{1}, \ldots, a_{d}\right\}$ be a finite, non-empty set. The elements of $D$ are referred to as dimensions. With

$$
A_{D}^{f}
$$

we denote the set of finite non-empty sequences of elements of $D$ in which each dimension occurs at most once, and with $\ell(w)$ we denote the length of $w \in A_{D}^{f}$. Note that $A_{D}^{f}$ is 
$P^{a, v, b, v^{\prime}}\left(y_{1}, x_{1}, \ldots, x_{m}, y_{2}, z_{1}, \ldots, z_{n}\right)=P^{b, v, a, v^{\prime}}\left(y_{2}, x_{1}, \ldots, x_{m}, y_{1}, z_{1}, \ldots, z_{n}\right)$

for all $a, b \in D$ and $\left(a, v, b, v^{\prime}\right) \in W$, where $v$ and/or $v^{\prime}$ can be empty ( $m=0$ and/or $\left.n=0\right)$

$$
\begin{aligned}
P^{a}(\top) & =1 \\
P^{a, v}\left(\top, x_{1}, \ldots, x_{k+1}\right) & =P^{v}\left(x_{1}, \ldots, x_{k+1}\right) \\
P^{w}(\perp, \vec{x}) & =0 \\
P^{w}(y, \vec{x}) & =\left|P^{w}(y, \vec{x})\right| \\
P^{w}(y \vee z, \vec{x}) & =P^{w}(y, \vec{x})+P^{w}(z, \vec{x})-P^{w}(y \wedge z, \vec{x})
\end{aligned}
$$

Table 6: $P F F_{W, P}$, axioms for a PFF with arity family $W$ and name $P$, where $a \in D, k \in \mathbb{N}$, $\vec{x}=x_{1}, \ldots, x_{k}$ and $P(y, \vec{x})=P(y)$ if $k=0$, and $w=(a, u) \in W$ with $\ell(w)=k+1$

finite. Elements of $A_{D}^{f}$ serve as arities of probability functions on a multi-dimensional event space of dimension $\ell(w)$. If $\ell(w)>1$, then $w$ is written as a comma-separated sequence, e.g. $\ell\left(a_{1}, a_{3}\right)=2$ and we write $\left(a_{1}, a_{3}\right) \in A_{D}^{f}$.

Given an event space $E$ and a name $P$ for a probability function, an arity family for $D$ is a subset $W$ of $A_{D}^{f}$ that is closed under permutation and under taking non-empty subsequences. Given an arity family $W$ for $D$, a function family for $W$ consists of a function $P^{w}: E^{\ell(w)} \rightarrow V$ for each arity $w \in W$. A function family for dimension set $D$, arity family $W$ and function name $P$ is a probability function family (PFF) if it satisfies the axioms of Table 6. Because in an arity repetition of dimensions is disallowed, these axioms reduce to what we had already in the case of a single dimension.

\subsection{Existence of a universal probability function}

A subset $W$ of $A_{D}^{f}$ may or may not have a maximal element under inclusion. If $W$ has a maximal element $\bar{w}$ and if we have a probability function family $\left(P^{w}\right)_{w \in W}$ for $W$, then $P^{\bar{w}}$ serves as a universal element for the family of probability functions because all other members of it can be found via successive application of the axioms (27) - (30).

As it turns out some PFFs cannot be extended with a universal PF. In the notation of our specification of probability families we will state a specific result that may serve as a necessary condition for the possibility to extend a PFF with a universal element.

Theorem 5.2.1. Given a set of dimensions $D=\{a, b, c, d\}$, an arity family $W$ for $D$ that satisfies $W \supset\{(b, c),(b, d),(a, d),(a, c)\}$, and a PFF $\left(P^{w}\right)_{w \in W}$, let $t$ be the following term:

$$
t=P^{b, c}(y, z)+P^{b, d}(y, u)+P^{a, d}(x, u)-P^{a, c}(x, z)-P^{b}(y)-P^{d}(u) .
$$

Then, if $W$ has a maximal element, then $-1 \leq t \leq 0$, that is, the following two inequalities must hold for $G_{W, P}=B A+M d+\operatorname{Sign}+P F F_{W, P}$ :

$$
G_{W, P} \vdash t+1=\mathbf{s}(t+1) \cdot(t+1) \quad \text { and } \quad G_{W, P} \vdash-t=\mathbf{s}(-t) \cdot-t .
$$


Clearly if a PFF for $D$ contains all of $P^{b, c}, P^{b, d}, P^{a, d}, P^{a, c}$ and if it fails to meet either one of the mentioned inequalities on $t$, then a universal $\mathrm{PF}$ cannot be found for it.

These facts are known as the BCHS (Bell, Clauser, Horne, Shimony) inequalities. Both were formulated and shown in a set theoretic framework for probability theory in [14] and [10, and a straightforward proof is given in [12, Section 9.2] 9 which we repeat here.

Proof of Theorem 5.2.1 (taken from [12]).

$$
\begin{aligned}
P^{b, c, d}(y, z, u) & =P^{a, b, c, d}(x, y, z, u)+P^{a, b, c, d}(\neg x, y, z, u) \\
& \leq P^{a, c}(x, z)+P^{a, d}(\neg x, u) \\
& =P^{a, c}(x, z)+P^{d}(u)-P^{a, d}(x, u), \\
P^{b, c, d}(\neg y, z, u) & =P^{a, b, c, d}(x, \neg y, z, u)+P^{a, b, c, d}(\neg x, \neg y, z, u) \\
& \leq P^{a, d}(x, u)+P^{a, c}(\neg x, z) \\
& =P^{a, d}(x, u)+P^{c}(z)-P^{a, c}(x, z), \\
0 & \leq P^{b, c, d}(y, \neg z, \neg u) \\
& =P^{b, c}(y, \neg z)-P^{b, c, d}(y, \neg z, u) \\
& =P^{b}(y)-P^{b, c}(y, z)-P^{b, d}(y, u)+P^{b, c, d}(y, z, u) .
\end{aligned}
$$

Combining (33) and (35) yields

$$
0 \leq P^{b}(y)-P^{b, c}(y, z)-P^{b, d}(y, u)+P^{a, c}(x, z)+P^{d}(u)-P^{a, d}(x, u) .
$$

From (35) and the equality

$$
-P^{c, d}(z, u)+P^{c, d}(\neg z, \neg u)=1-P^{c}(z)-P^{d}(u)
$$

it follows that

$$
\begin{aligned}
0 & \leq P^{b, c, d}(\neg y, \neg z, \neg u) \\
& =P^{c, d}(\neg z, \neg u)-P^{b, c, d}(y, \neg z, \neg u) \\
& =1-P^{b}(y)-P^{c}(z)-P^{d}(u)+P^{b, c}(y, z)+P^{b, d}(y, u)+P^{b, c, d}(\neg y, z, u) .
\end{aligned}
$$

Then from (34) and (37) we get

$$
0 \leq 1-P^{b}(y)-P^{d}(u)+P^{b, c}(y, z)+P^{b, d}(y, u)+P^{a, d}(x, u)-P^{a, c}(x, z) .
$$

Inequalities (36) and (38) can be combined to give the inequalities of the theorem.

\footnotetext{
${ }^{9}$ From this pair of inequalities one can derive the original Bell inequalities from 3. The key observation of Bell was that quantum mechanics gives rise to the hypothesis that a four-dimensional event space exists in which a family of joint probabilities for at most two dimensions can be found that violates the inequalities from the theorem.
} 


\subsection{The multi-dimensional case and the one-dimensional case}

The one-dimensional case is obtained from the multi-dimensional case by taking for $D$ a singleton set, say $D=\{d\}$, and subsequently forgetting the single name involved. The more challenging question then arises if the multi-dimensional case is already implicit in the onedimensional case. The objective of this section is to discuss that matter in some detail.

Some additional terminology will be needed. A permutation compatible function family for function name $P$ and an arity family $W$ is a function family for $P$ and $W$ that satisfies the permutation axiom (27) in Table 6.

Given a compatible function family $F_{P, W}$ for $P$ and $W$ its hull of one-dimensional projections is defined as the collection of functions $Q: E \rightarrow V$ each of which can be obtained from some $P^{w}: E^{\ell(w)} \rightarrow V$ in $F_{P, W}$ for some $w=\left(d, u_{1}, \ldots, u_{t}\right) \in W$ by choosing $e_{1}, \ldots, e_{t}$ in $E^{t}$ and by setting $Q(x)=P\left(x, e_{1}, \ldots, e_{t}\right)$.

We may now look at the specification $B A+M d+\operatorname{Sign}+P F_{P}$ in a different way: it axiomatizes the notion of a probability function rather than of a particular structure with a probability function. Then one may define a multi-dimensional PFF (for $P$ and $W$ ) alternatively as a compatible function family such that each function in its one-dimensional hull is a probability function according to the axioms of $B A+M d+S i g n+P F_{P}$.

This is as close as we can get in turning the multi-dimensional case into an application of the one-dimensional case. It is not a formal reduction because that would require that it is guaranteed that for each $Q$ the same meadow is used to determine its status as a probability function. The latter constraint lies outside the expressive power of first order equational logic, however. This leaves us with the following state of affairs: although $B A+M d+\operatorname{Sign}+$ $P F_{P}$ axiomatizes probability functions in a satisfactory manner, when it comes to essential allocations such as Theorem 5.2.1, a more general axiom system is needed to take care of the multi-dimensional case.

\section{Concluding remarks}

The incentive for this work came from a talk given by professor Ian Evett on the occasion of the retirement of dr. Huub Hardy as a driving force behind the MSc Forensic Science at the University of Amsterdam 10 That talk illustrated the headway that the Bayesian approach to reasoning in forensic matters has made in recent years. However, Evett also highlighted the conceptual and political problems that may still lie ahead of its universal adoption in the legal process.

In order to improve the understanding of these issues an elementary logical formalization of reasoning with probabilities might be useful. With that perspective in mind we came to the conclusion that in spite of the abundance of introductory texts to probability theory, the development of an axiomatic approach from first principles may yet cover new ground. The formalization of probabilities in terms of equational logic outlined above is intended to serve as a point of departure from which to develop presentations of probability theory that may be be helpful when a formal and logically precise perspective on reasoning with probabilities is aimed at.

\footnotetext{
${ }^{10}$ This meeting took place at Science Park Amsterdam, Friday June 7, 2013 under the heading "Frontiers of Forensic Science", and was organized by Andrea Haker.
} 
We acknowledge many discussions with Andrea Haker (University of Amsterdam) regarding the relevance of logically grounded reasoning methodologies in forensic science.

\section{References}

[1] E. Adams. Probability and the logic of conditionals. In: J. Hintikka and P. Suppes (eds.), Aspects of Inductive Logic, North-Holland, 265-316, 1966.

[2] David Barber. Bayesian Reasoning and Machine Learning. Cambridge University Press, 2012. (ISBN 0521518148, 9780521518147). On-line version (18 November 2015) available: http://web4.cs.ucl.ac.uk/staff/D.Barber/pmwiki/pmwiki.php?n=Brml.Online.

[3] J.S. Bell. On the Einstein Podolsky Rosen paradox. Physics, 1(3):195-200,1964.

[4] J.A. Bergstra, I. Bethke, and A. Ponse. Cancellation meadows: a generic basis theorem and some applications. The Computer Journal, $56(1): 3-14, \quad 2013$ (doi.org/10.1093/comjnl/bxs028). Also available at https://arxiv.org/abs/0803.3969v3

[5] J.A. Bergstra, I. Bethke, and A. Ponse. Equations for formally real meadows. Journal of Applied Logic, 13(2) part B:1-23, 2015 (doi.org/10.1016/j.jal.2015.01.004). Also available at https://arxiv.org/abs/1310.5011v4

[6] J.A. Bergstra and C.A. Middelburg. Inversive meadows and divisive meadows. Journal of Applied Logic, 9(3): 203-220, 2011. Also available at https://arxiv.org/abs/0907.0540v3

[7] J.A. Bergstra and C.A. Middelburg. Division by zero in involutive meadows. Journal of Applied Logic, 13(1): 1-12, 2015. Also available at https://arxiv.org/abs/1406.2092

[8] J.A. Bergstra and A. Ponse. Fracpairs and fractions over a reduced commutative ring. Indagationes Mathematicae, 27:727-748, 2016 dx.doi.org/10.1016/j.indag.2016.01.007 Also available at https://arxiv.org/abs/1411.4410v2

[9] J. Carlström. Wheels - on division by zero. Mathematical Structures in Computer Science, 14(1):143-184, 2004 doi:10.1017/S0960129503004110

[10] A. Fine. Hidden variables, joint probability, and the Bell inequalities. Physical Review Letters, 48(5):291-295, 1982 (doi.org/10.1103/PhysRevLett.48.291).

[11] P. Milne. Bruno de Finetti and the logic of conditional events. Brit. J. Phil. Sci, 48:195232,1997

[12] W.M. de Muynck. Foundations of quantum mechanics, an empiricist approach. Fundamental theories of physics, Vol. 127. Kluwer Academic Publishers, 2002. Pre-publication version: http://www.phys.tue.nl/ktn/Wim/Kluwerbookprepversion.pdf

[13] H. Padmanabhan. A self-dual equational basis for Boolean algebras. Canadian Mathematical Bulletin, 26(1):9-12, 1983.

[14] P. Rastall. The Bell inequalities. Foundations of Physics, 13(6):555-570, 1983. 
[15] Tiago S. dos Reis, Walter Gomide, and James A.D.W. Anderson. Construction of the Transreal Numbers and Algebraic Transfields. IAENG International Journal of Applied Mathematics, 46(1):11-23, 2016. Available at http://www.iaeng.org/IJAM/issues_v46/issue_1/IJAM_46_1_03.pdf

\section{A A standard example of equational probabilistic rea- soning}

In this appendix we analyse a straightforward example of an application of Bayes' theorem. This example has been derived from Example 1.2 as presented in the freely accessible 2015version of [2. In Appendix A.1 the example is described, and in Appendix A.2 we analyse how a simple modification of this example may turn it into an inconsistent one and draw some conclusions.

\section{A.1 The example}

We assume the following hypothetical but conceivable data:

1. A rare disease RD occurs with probability $1 / 100,000$ in the population of a country CO.

2. A potentially problematic nutritional habit $\mathrm{NH}$ is very widespread, in fact 4 out of 10 people in $\mathrm{CO}$ show $\mathrm{NH}$.

3. It has been found that 8 out of 10 persons in $\mathrm{CO}$ who are suffering from $\mathrm{RD}$ show $\mathrm{NH}$ as well.

The question is to find the probability that someone showing $\mathrm{NH}$ suffers from $\mathrm{RD}$. In order to answer that question the formalization of the three facts is as follows: we assume that RD and $\mathrm{NH}$ are names for events, and we assume that the PF $P$ comprises the available probabilistic data: $P(\mathrm{RD})=1 / 100,000, P(\mathrm{NH})=4 / 10$, and $P^{0}(\mathrm{NH} \mid \mathrm{RD})=8 / 10$.

Strictly speaking RD and NH are used as event variables and the assumptions are viewed as conditions. Computing a probability is used as a shorthand for proving that it equals some real value.

The question then is to compute $p=P^{0}(\mathrm{RD} \mid \mathrm{NH})$. Using Bayes' theorem one finds:

$$
p=\frac{P^{0}(\mathrm{NH} \mid \mathrm{RD}) \cdot P(\mathrm{RD})}{P(\mathrm{NH})}=\frac{8 / 10 \cdot 1 / 100,000}{4 / 10}=0.2 \cdot 10^{-4} .
$$

Thus, we find for $E=\left\{P(\mathrm{RD})=1 / 100,000, P(\mathrm{NH})=4 / 10, P^{0}(\mathrm{NH} \mid \mathrm{RD})=8 / 10\right\}$ that

$$
B A+M d+\operatorname{Sign}+P F_{P}+E \vdash P^{0}(\mathrm{RD} \mid \mathrm{NH})=0.2 \cdot 10^{-4} .
$$

At face value this result is informative. 


\section{A.2 Modifications of the example}

One may notice that if $\mathrm{NH}$ were less widespread, say $P(\mathrm{NH})=1 / 500$, the value of $P^{0}(\mathrm{RD} \mid \mathrm{NH})$ computed above changes significantly: in this case we find for $q=P^{0}(\mathrm{RD} \mid \mathrm{NH})$ that

$$
q=\frac{P^{0}(\mathrm{NH} \mid \mathrm{RD}) \cdot P(\mathrm{RD})}{P(\mathrm{NH})}=\frac{8 / 10 \cdot 1 / 100,000}{1 / 500}=0.4 \cdot 10^{-2} .
$$

Again, at face value this result is informative.

Now we may consider the case that the occurrence of $\mathrm{NH}$ is even more rare, say 1 out of $1,000,000$. For $r=P^{0}(\mathrm{RD} \mid \mathrm{NH})$ we find in this case

$$
r=\frac{P^{0}(\mathrm{NH} \mid \mathrm{RD}) \cdot P(\mathrm{RD})}{P(\mathrm{NH})}=\frac{8 / 10 \cdot 1 / 100,000}{1 / 1,000,000}=8 .
$$

This outcome is of course "wrong" (probabilities are supposed not to exceed 1).

The interesting aspect of this example and its modifications is that the first two equations and computations (for $p$ and for $q$ ) correspond with conventional textbook examples, while the third variation (for $r$ ) indicates that something might have gone wrong in all three cases. We conclude this:

1. The production of a value for $P^{0}(\mathrm{RD} \mid \mathrm{NH})$ that exceeds 1 constitutes a failure of the reasoning process at hand.

2. That failure is caused by an underlying fault (the failure is merely a symptom of that fault).

3. The failure lies in non-detection of the fact that the third set of assumptions is incoherent: it represents a specification of a partial function from $E$ to $\mathbb{R}_{0}$ which cannot be extended to a total PF. In this case, with $E=\left\{P(\mathrm{RD})=10^{-5}, P(\mathrm{NH})=10^{-6}, P^{0}(\mathrm{NH} \mid \mathrm{RD})=\right.$ $\left.8 \cdot 10^{-1}\right\}$ :

$$
B A+M d+\operatorname{Sign}+P F_{P}+E \vdash 0=1 .
$$

To see this one may notice that the data from which $P^{0}(\mathrm{RD} \mid \mathrm{NH})$ has been computed allow the following proof:

$$
1 / 1,000,000=P(\mathrm{NH}) \geq P(\mathrm{NH} \wedge \mathrm{RD})=P^{0}(\mathrm{NH} \mid \mathrm{RD}) \cdot P(\mathrm{RD})=8 / 10 \cdot 1 / 100,000
$$

from which one easily obtains $0=1$.

4. Determining the fault underlying the failure is harder. When providing an example either a coherence check should have been be applied to the original data, or the risk of getting invalid results must be accepted. Both assertions lie outside equational logic proper. As the consistency check is an NP-complete question in general the suggestion to check consistency first may be considered unconvincing.

5. Assuming that data come from valid experimental procedures, the realistic background of these data may be understood to provide a justification for not checking consistency in advance of further usage. Under that assumption calculations involving Bayes' theorem such as in the example are justified. 